\title{
A Sinorhizobium meliloti Lipopolysaccharide Mutant Induces Effective Nodules on the Host Plant Medicago sativa (Alfalfa) but Fails to Establish a Symbiosis with Medicago truncatula
}

\author{
K. Niehaus, ${ }^{1}$ A. Lagares, ${ }^{2}$ and A. Pühler ${ }^{1}$ \\ ${ }^{1}$ Universität Bielefeld, Fakultät für Biologie, Lehrstuhl für Genetik, Postfach 100131, D-33501 Bielefeld, \\ Germany; ${ }^{2}$ Cátedra de Quimica I, Facultad de Ciencias Exactas, Universidad Nacional de La Plata, \\ 47 y 115, 1900-La Plata, Argentina \\ Accepted 25 May 1998.
}

The specific Sinorhizobium meliloti lipopolysaccharide (LPS) mutant Rm6963 (A. Lagares, G. Caetano Anolles, K. Niehaus, J. Lorenzen, H. D. Ljunggren, A. Puhler, and G. Favelukes, J. Bacteriol. 174:5941-5952, 1992) was shown to be mutated in a region corresponding to a cloned 5-kb SstI DNA fragment that was able to complement the lps B and lps $\mathrm{C}$ mutants of $S$. meliloti described by Clover et al. (R. H. Clover, J. Kieber, and E. R. Signer, J. Bacteriol. 171:3961-3967, 1989). Sodium dodecyl sulfate polyacrylamide electrophoresis revealed that the LPS-I and LPS-II fractions of the LPS mutant Rm6963 were shifted to lower molecular weights. While the majority of the Medicago spp. tested established an effective symbiosis with both the $S$. meliloti wild-type $R \mathbf{m} 2011$ and the LPS mutant Rm6963, the latter induced ineffective nodules on $M$. truncatula. A light- and electron-microscopic analysis of the ineffective $M$. truncatula root nodules revealed that the bacteria were released from the infection threads but failed to colonize the plant cells effectively. The plant cytoplasm was filled with numerous vesicles, probably the result of a disturbed bacteroid development. Sections of ineffective $M$. truncatula root nodules induced by the LPS mutant Rm6963 showed brown, necrotic cells within the central nodule tissue that autofluoresced when viewed under UV light. These observations are best explained by a plant defense response. Evidently, the rhizobial LPS plays a role in plant-microbe signaling during the formation of M. truncatula nodules.

The soil bacterium Sinorhizobium meliloti is well known for its capacity to form root nodules with the leguminous plants of the genera Medicago, Melilotus, and Trigonella. By mutational analysis, different sets of $S$. meliloti genes were identified that play a role in nodulation and nitrogen fixation (reviewed by Brewin 1991 and Hirsch 1992). A specific question concerns the involvement of the bacterial

Corresponding author: K. Niehaus; Telephone:+48 521106 5632; Fax: +48 521106 5626; E-mail: kniehaus@genetik.uni-bielefeld.de envelope in this interaction process. It was found that the secreted exopolysaccharide (EPS) succinoglycan EPS I plays an important role in the infection of alfalfa nodules by S. meliloti (Leigh and Coplin 1992). S. meliloti mutants that were blocked in the synthesis of EPS I failed to infect the plant. Nevertheless, these strains were able to induce noninfected pseudonodules (Finan et al. 1985; Müller et al. 1988). We observed plant defense responses in these pseudonodules, and postulated that in the wild-type alfalfa nodules these plant defense responses are suppressed by the rhizobial EPS (Niehaus et al. 1993).

In this paper, we report on the question whether the bacterial lipopolysaccharide (LPS) plays a role in the interaction of $S$. meliloti with its different host plants. For other symbiotic systems it has already been demonstrated that LPS plays an essential role. $R$. leguminosarum bvs. phaseoli, viciae, and trifolii and Bradyrhizobium japonicum mutants deficient in the biosynthesis of LPS were impaired in symbiosis (Noel 1992; Kannenberg and Brewin 1994). Rhizobium mutants carrying major modifications in the LPS molecule were no longer able to establish normal, nitrogenfixing nodules. LPS mutants of $R$. leguminosarum bvs. trifolii and viciae infected the root nodules, but rhizobial cells released from the infection thread failed to differentiate into normal, nitrogen-fixing bacteroids (Priefer 1989). LPS mutants of $R$. leguminosarum bv. phaseoli and B. japonicum were blocked even earlier in the interaction with the plant. These mutants were arrested in the development of infection threads, leading to noninfected pseudonodules (Carlson et al. 1987; Stacey et al. 1991). In contrast, S. meliloti LPS mutants formed nitrogen-fixing nodules on $M$. sativa (Clover et al. 1989). A detailed analysis of a specific $S$. meliloti LPS mutant, designated Rm6963, showed that the alfalfa nodulation competence of this mutant strain is strongly reduced, compared with the $S$. meliloti wild-type strain (Lagares et al. 1992).

In the present study we analyze the symbiotic properties of the above-mentioned $S$. meliloti LPS mutant Rm6963, and show that this mutant forms effective nodules with Medicago sativa but ineffective ones with $M$. truncatula. 


\section{RESULTS}

\section{Genetic and phenotypic analysis of the $S$. meliloti LPS mutant Rm6963.}

Following a general Tn5 mutagenesis of the $S$. meliloti wild-type strain Rm2011 the LPS mutant Rm6963 was identified due to a rough colony morphology and increased sensitivity to the detergent sodium dodecyl sulfate (SDS) (Lagares et al. 1992). For a phenotypic analysis of the LPS produced by the S. meliloti wild-type $\mathrm{Rm} 2011$ and the LPS mutant Rm6963, SDS-polyacrylamide gel electrophoresis (PAGE) was applied. With the increased resolution of the tricine-SDSpolyacrylamide system, the LPS of the different strains was examined and compared with Salmonella typhimurium LPS samples. After electrophoresis, typical banding patterns were obtained (Fig. 1). As described for other Rhizobium spp. (Carlson and Krishnaiah 1992), two forms of LPS were found for the S. meliloti strain Rm2011. The S. meliloti Rm2011 LPS-II showed a faster migration than that of LPS-I. The migration of LPS-II is comparable to that of the S. typhimurium Ra-mutant LPS (Fig. 1, lane 3), indicating that this band most probably represents lipid A and the core region. The slowmigrating LPS-I form showed a low degree of microheterogeneity. Only two bands could be detected. A ladderlike structure, as in the case of S. typhimurium LPS (Fig. 1, lane 1), was not observed. However, when the LPSs of $S$. typhimurium and $S$. meliloti $\mathrm{Rm} 2011$ were compared, it was concluded that the two LPS-I bands, LPS-Ia and LPS-Ib (Fig. 1, lane 4), may represent the entire LPS including the O-antigen. The mutant LPS of strain Rm6963 nearly resembles the wild-type LPS banding pattern, but shifted to a lower molecular weight (Fig. 1, lane 5). The fast-migrating LPS-II of the mutant strain Rm6963 was comparable to the migration of the LPS from the $S$. typhimurium Re-mutant. In Salmonella spp. this fast migrating molecule was identified as lipid A substituted with KDO (Hitchcock and Brown 1983). Both LPS-I bands were present; however, LPS-Ia' was hardly visible. The appearance of LPS-I was surprising, since the faster migration of LPS-II indicates a mutation within the LPS core region, which should cause a total loss of the $\mathrm{O}$-antigen that is believed to be a part of LPS-I. Since the biochemical function of the mutated gene is not known, surface carbohydrates other than the LPS may be affected. Nevertheless, the amount and the monosaccharide composition of the major EPS, EPS I, is not altered (data not shown).

General transduction with phage $\phi \mathrm{M} 12$ showed that the neomycin resistance of the Tn5 in strain Rm6963 was always co-transducible with the sensitivity to SDS (Lagares et al. 1992). In order to analyze the mutated DNA region of the strain Rm6963 more precisely, the plasmid pJL201 carrying the Tn5-mutation on a 5-kb SstI DNA fragment was constructed. To prove that the Tn5 insertion in Rm6963 caused the LPS mutation, the mutated 5-kb SstI DNA fragment was used for a homogenotization experiment of the wild-type strain Rm2011. Gene replacement was achieved by antibiotic selection for double recombination events after introduction of pJL201 into Rm2011. The obtained Tn5 mutant, termed RmJL1, showed the same LPS phenotype as the original mutant Rm6963 (Fig. 1, lanes 5 and 6). In a further experiment plasmid pAL101 carrying the wild-type 5-kb SstI DNA fragment was isolated again by gene replacement. After pAL101 was introduced into the mutant strain Rm6963, the resulting transconjugants showed the same LPS banding pattern as the wild-type Rm2011 (Fig. 1, lanes 4 and 7).

Restriction analysis of the 5-kb SstI fragment (data not shown) gave evidence that the analyzed fragment is part of the lps $\mathrm{B} /$ lps $\mathrm{C}$ region published by Clover et al. (1989). For this reason pJL201, carrying the mutated 5-kb SstI fragment, was introduced into the $S$. meliloti mutants $\mathrm{Rm} 7670$ (lpsB::Tn5) and $\mathrm{Rm} 7555$ (lpsC::Tn5). The resulting transconjugants were analyzed for their LPS phenotype by SDS-PAGE. The lpsC mutant but not the lpsB mutant was complemented by pJL201. Since both mutants were complemented by pAL101 carrying the native 5-kb Sst I fragment it can be concluded that Rm6963 carries a mutation in the $\operatorname{lps} \mathrm{B}$ region.

\section{The $S$. meliloti LPS mutant Rm6963 formed an effective symbiosis with $M$. sativa but was ineffective with M. truncatula.}

The S. meliloti LPS mutant Rm6963 showed delayed nodulation, but established nitrogen-fixing nodules on $M$. sativa (Lagares et al. 1992). Since a defect in symbiosis was described for LPS mutants of $R$. leguminosarum and other Rhizobium spp., we studied the interaction of the $S$. meliloti LPS mutant Rm6963 with different host plants. All tested Medicago spp. were grown on nitrogen-free agar plates and inoculated with the wild-type strain Rm2011 or the LPS mutant strain Rm6963. Three weeks after inoculation the plants were assayed for acetylene reduction as a measure for an effective symbiosis. A small group of Medicago spp. showed an ineffective symbiosis with both the $S$. meliloti wild-type or the mutant strain (Table 1). The majority of the Medicago spp. established a nitrogen-fixing symbiosis with both the wildtype Rm2011 and the mutant Rm6963 (Table 1). Interestingly, a small group of species, including $M$. truncatula, established a normal, effective symbiosis with $S$. meliloti Rm2011, but failed

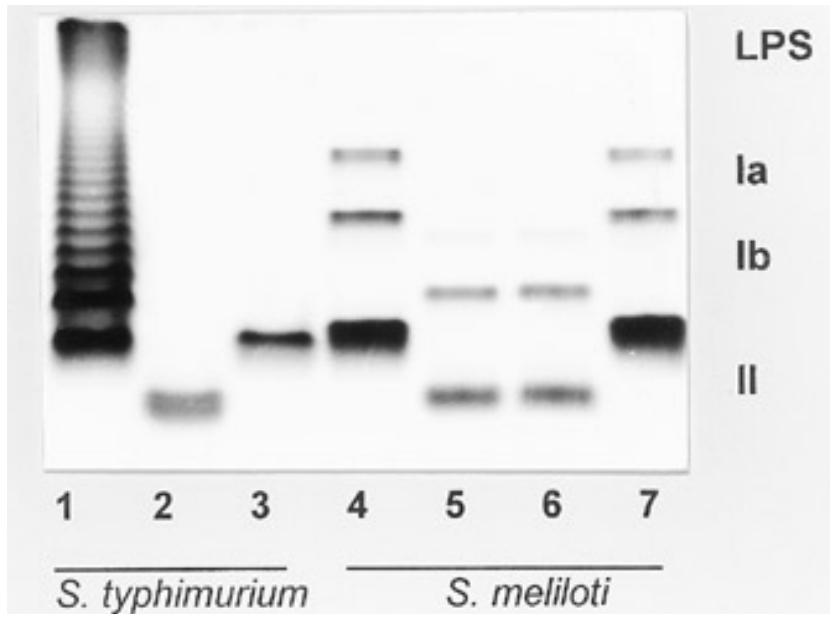

Fig. 1. Lipopolysaccharide (LPS) profiles of Sinorhizobium meliloti wild-type and mutant strains following polyacrylamide gel electrophoresis and silver staining. LPS profiles of the S. meliloti wild-type strain Rm2011 (lane 4) and the LPS mutants Rm6963 (lane 5), RmJL1 (lane 6), and Rm6963 complemented with the plasmid pAL101 (lane 7). LPSs isolated from the Salmonella typhimurium wild-type (lane 1), the S. typhimurium Re mutant (lane 2), and the S. typhimurium Ra mutant (lane 3) were used as standards. Positions of LPS-I and LPS-II are marked. Slow-migrating LPS-I was subdivided into LPS-Ia and LPS-Ib. LPS bands were shifted to a faster migration in the case of the mutants. 
to fix nitrogen when inoculated with the LPS mutant Rm6963 (Table 1). This defect was analyzed in detail for the widely used legume $M$. truncatula and compared with $M$. sativa.

In order to determine the nodulation kinetics, seedlings were transferred to petri dishes containing nitrogen-free agar. When $M$. sativa plants were inoculated with the $S$. meliloti wild-type strain $\mathrm{Rm} 2011$ the first visible nodules were observed after 6 days, while in plants inoculated with the LPS mutant Rm6963 the first nodules appeared only 3 to 4 days later (Fig. 2A). The same delay was observed in the onset of nitrogen fixation. Nevertheless, 3 weeks after inoculation, the nitrogen-fixing capacity of the nodules induced by the LPS mutant strain was even higher than of those induced by the wild-type strain (Fig. 2A). Four weeks after inoculation, the total nitrogen content of whole plants was determined. $M$. $s a$ tiva plants inoculated with $S$. meliloti $\mathrm{Rm} 2011$ or the mutant Rm6963 showed the same nitrogen content of $44 \pm 6 \mathrm{mg}$ of $\mathrm{N}$ per g of dry mass.

Light microscopic analyses of $M$. sativa nodules induced by Rm2011 or Rm6963 showed no difference, indicating that the defect of the LPS mutant is restricted to the early events of plant-microbe interaction. A delay in nodulation as observed in the $M$. sativa-Rm6963 interaction was also recognized after inoculation of $M$. truncatula with Rm6963. Plants inoculated with the LPS mutant were nodulated with a delay of 3 to 4 days, compared with those inoculated with Rm2011 (Fig. 2B). While $M$. truncatula plants inoculated with Rm2011 showed the onset of nitrogen fixation after 10 days, no nitrogen fixation was observed when plants were inoculated with Rm6963

Table 1. Analysis of the nitrogen fixation properties of different Medicago spp. after inoculation with the Sinorhizobium meliloti wild-type Rm2011 or the lipopolysaccharide-deficent mutant Rm6963

\begin{tabular}{|c|c|c|c|c|}
\hline \multirow[b]{3}{*}{ Plant species } & \multicolumn{4}{|c|}{ S. meliloti } \\
\hline & \multicolumn{2}{|c|}{ Rm2011 } & \multicolumn{2}{|c|}{ Rm6963 } \\
\hline & Nod. & Fix. & Nod. & Fix. \\
\hline M. carstinensis & + & - & + & - \\
\hline M. ciliaris & + & - & + & - \\
\hline M. coronata & - & - & - & - \\
\hline M. glandulosa & + & - & + & - \\
\hline M. intertexta var. intertexta & + & - & + & - \\
\hline M. nigra var. brevisina & + & - & + & - \\
\hline M. nigra var. vulgaris & + & - & + & - \\
\hline M. rugosa & - & - & - & - \\
\hline M. cancellata & + & + & + & + \\
\hline M. disciformis & + & + & + & + \\
\hline M. glutinosa subsp. polychroa & + & + & + & + \\
\hline M. murex var. murex & + & + & + & + \\
\hline M. orbicularis & + & + & + & + \\
\hline M. romanica & + & + & + & + \\
\hline M. sativa subsp. caerulea & + & + & + & + \\
\hline M. sativa subsp. sativa & + & + & + & + \\
\hline M. sativa $\mathrm{cv}$. Du Puits & + & + & + & + \\
\hline M. sativa $\mathrm{cv}$. Chinskeja Mestnaja & + & + & + & + \\
\hline M. sativa $\mathrm{cv}$. Europe & + & + & + & + \\
\hline M. sativa $\mathrm{cv}$. Hairy Peruvian & + & + & + & + \\
\hline M. sativa $\mathrm{cv}$. Normad & + & + & + & + \\
\hline M. varia $\times$ varia & + & + & + & + \\
\hline M. blancheana var. blancheana & + & + & + & - \\
\hline M. truncatula & + & + & + & - \\
\hline M. truncatula var. longispinia & + & + & + & - \\
\hline
\end{tabular}

${ }^{a}$ Nod: nodulation of test plant; Fix: nitrogen fixation ability of root nodules. + , effective nodulation and nitrogen fixation;,- , no nodulation or no nitrogen fixation.
(Fig. 2B). Also, after a prolonged incubation (5 weeks) of the $M$. truncatula plants with the LPS mutant, no nitrogen fixation was detectable. The $S$. meliloti LPS mutant RmJL1, constructed by site-specific mutagenesis with the Tn5-labeled, 5kb SstI DNA fragment, was also unable to establish a nitrogen-fixing symbiosis in combination with M. truncatula, conferring the results obtained with the LPS mutant Rm6963.

\section{M. truncatula nodules induced by the $S$. meliloti LPS} mutant Rm6963 were disturbed in bacteroid development.

Root nodules of $M$. truncatula induced by the $S$. meliloti wild-type and the LPS mutant Rm6963 were examined by light- and electron-microscopic techniques to define the developmental steps at which nodule development was blocked. Within 3 weeks after inoculation with the $S$. meliloti wild-type
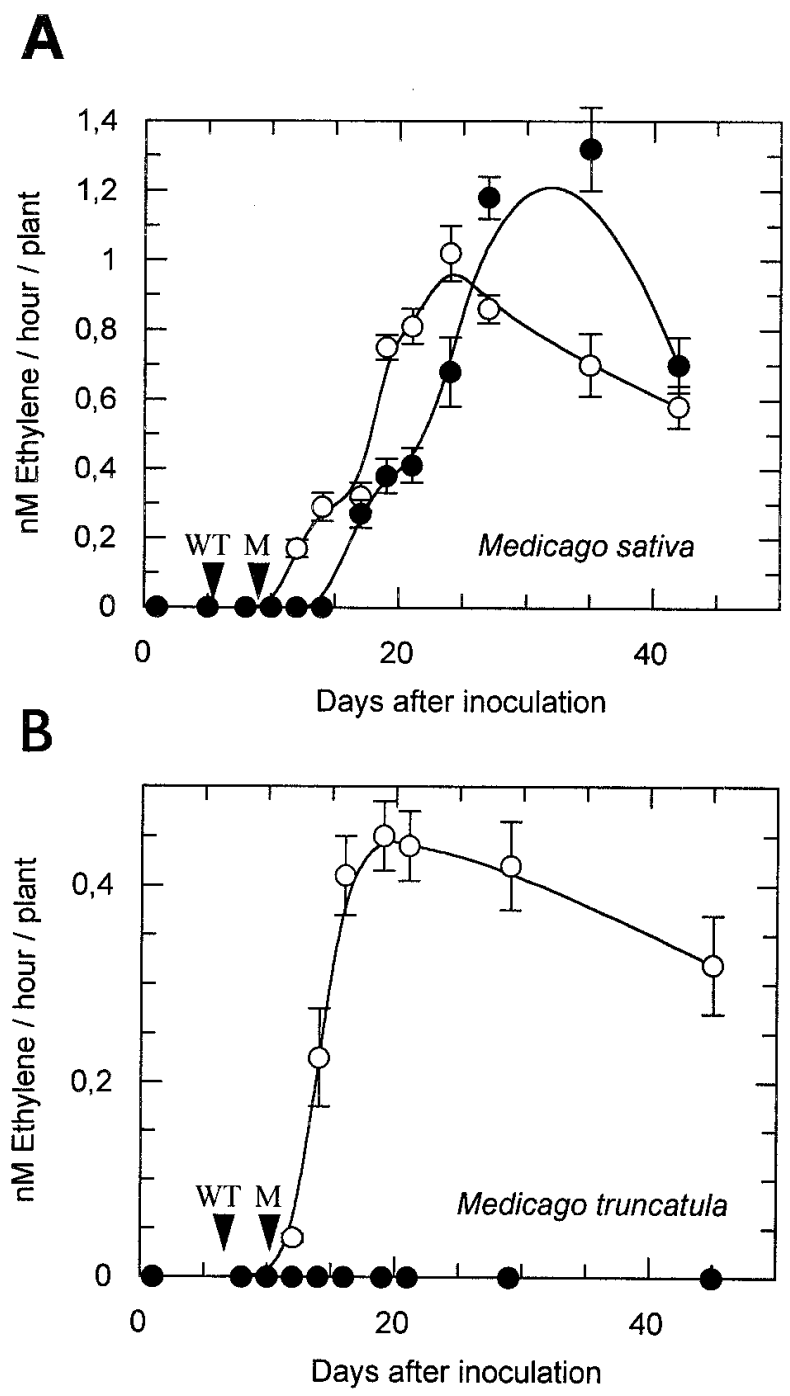

Fig. 2. Acetyle reduction of Medicago sativa and M. truncatula root nodules induced by the Sinorhizobium meliloti wild-type strain Rm2011 or the S. meliloti LPS mutant Rm6963. A, M. sativa was inoculated with the $S$. meliloti wild-type strain Rm2011 (open circles) and the LPS mutant Rm6963 (black circles). B, Same experimental setup as A, but $M$. sativa was replaced by $M$. truncatula. Each value is the mean of 10 determinations. First appearance of root nodules induced by the wild-type Rm2011 (WT) and the mutant Rm6963 (M) is marked. 
strain Rm2011, all M. truncatula plants developed cylindrical nodules exhibiting a light pink color due to their leghemoglobin content. Semi-thin sections of these nodules revealed the typical zonation of indeterminate root nodules with an apical meristem, an infection zone, a symbiotic zone, and a small senescent zone. After release from the infection thread, bacteria multiplied within the plant cells, giving rise to the fully infected cells of the symbiotic zone (Fig. 3A). The LPSdeficient S. meliloti mutant Rm6963, on the other hand, induced smaller cylindrical nodules, lacking the typical red color of leghemoglobin. A detailed analysis of 3-week-old nodules induced by Rm6963 resulted in the detection of poorly infected plant cells (Fig. 3B). The fully infected plant cells typical for the symbiotic zone of a nitrogen-fixing nodule were missing. A remarkable structural feature of these nodules induced by the LPS mutant when inoculated on $M$. truncatula was the enlarged infection threads. The wild-type, induced infection threads were two to four bacterial cells wide (Fig. 4A), while infection threads induced by the LPS mutant Rm6963 contained up to 20 rhizobial cells in diameter (Fig. 4B). Both infection threads contained the typical, gumlike thread matrix. At the ultrastructural level, the walls of the mutant-induced threads were less regularly formed and showed more fibrillar material. Rhizobia released from these infection threads differentiated morphologically into bacteroids (Fig. 5A). These bacteroids often showed a branched, irregular shape. The low density of bacteria per plant cell indicated a defect in the bacterial cell division. Interestingly, the cytoplasm of the infected plant cells was filled with numerous vesicles containing only little fibrillar material (Fig. 5B). These vesicles may have arisen due to a defect in the packaging of rhizobial cells within a peribacteroid membrane during their release from the infection threads. These vesicles were never observed in nodules infected by the wild-type strain Rm2011.

Histochemical changes in the central tissue were frequently observed in $M$. truncatula nodules induced by the mutant strain Rm6963. Fresh sections of root nodules showed brown, necrotic cells within the central nodule tissue (Fig. 6A). Fluorescence microscopy demonstrated, besides a nonspecific blue-white autofluorescence in the outer cells, an additional yellow and red fluorescence of some plant cells within the central nodule tissue (Fig. 6B), indicating the presence of phenolic compounds. A yellow or red autofluorescence of an infected plant was never observed after inoculation with the wild-type Rm2011.

\section{DISCUSSION}

The chemical structure and the genes responsible for the biosynthesis of LPS in S. meliloti Rm2011 are for the most part unknown. In this paper we show that at least one gene responsible for LPS biosynthesis is located on a $5-\mathrm{kb}$ SstI DNA fragment. It was interesting to find that this DNA fragment was able to complement the $\operatorname{lps} \mathrm{B}$ and $\operatorname{lps} \mathrm{C}$ genes described by Clover et al. (1989).

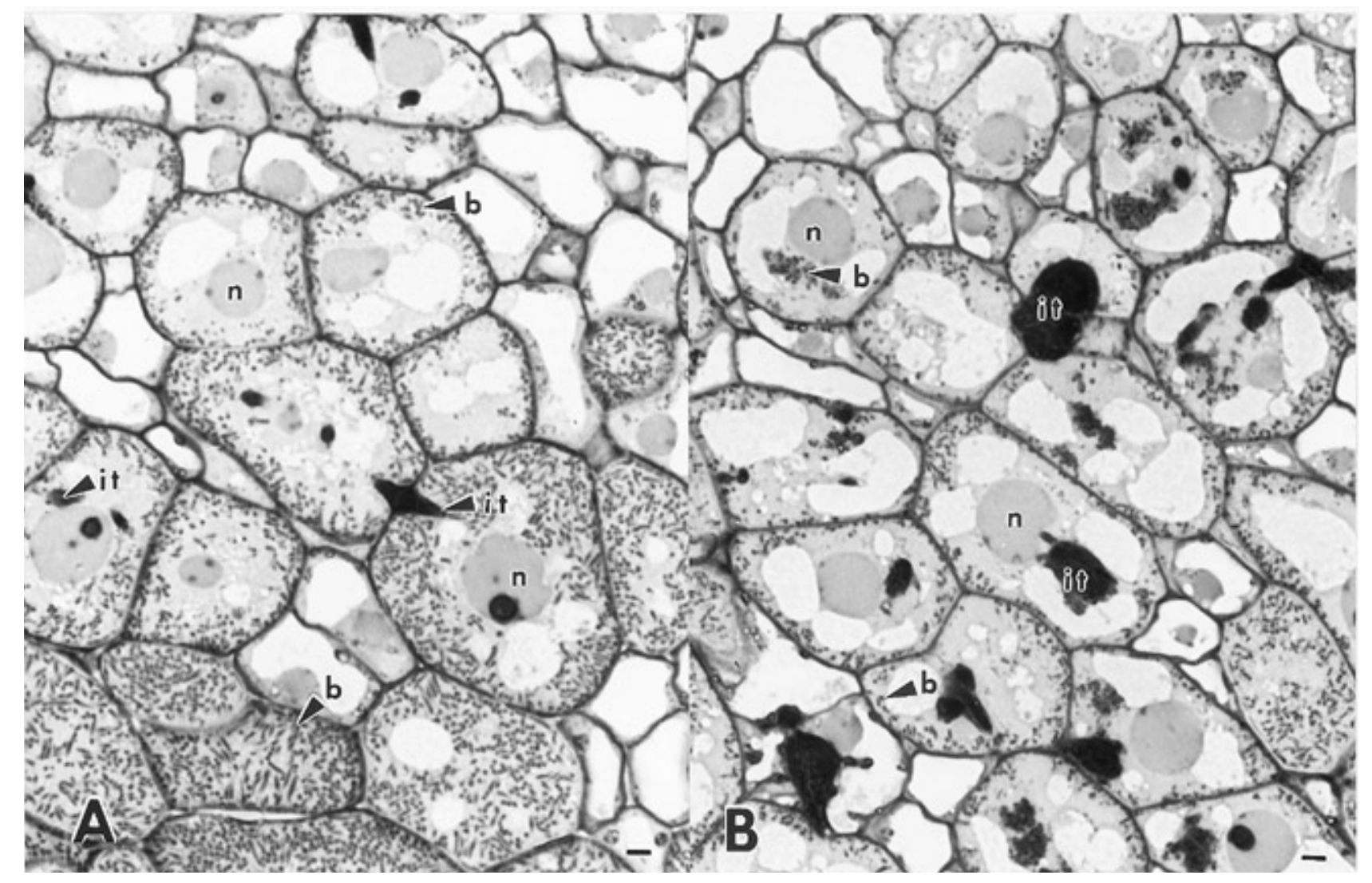

Fig. 3. Semi-thin sections of Medicago truncatula root nodules induced by (A) the Sinorhizobium meliloti wild-type Rm2011 and (B) the lipopolysaccharide (LPS)-deficient mutant Rm6963. Nodules were harvested 3 weeks after inoculation. Semi-thin sections were stained with toluidine blue O. b, bacteroids; it, infection thread; n, nuclei. Scale bar represents $5 \mu \mathrm{m}$. 
SDS-PAGE is a suitable tool for an initial characterization of the bacterial LPS (Hitchcock and Brown 1983). In this work we achieved a high resolution of the $S$. meliloti LPS pattern with the tricine-SDS-PAGE (Carlson 1984; Lesse et al. 1990). In comparison to previous experiments (Lagares et al. 1992), LPS-I could be separated into two well-defined bands, LPS-Ia and LPS-Ib. In the case of the LPS mutant Rm6963 the LPS pattern was shifted to a lower molecular weight. This behavior is of particular interest, because a faster migration of LPS-II indicates a mutation within the core oligosaccharide, normally resulting in the loss of the Oantigen represented by the LPS-I bands. In the case of the LPS mutant Rm6963 the migration behavior may indicate that the mutation led to a truncated core oligosaccharide represented by the fast-migrating LPS-II, and a truncated core connected to an intact O-antigen represented by the LPS-I bands. This explanation would imply that the core oligosaccharide has a branched structure, which was already reported for $R$. etli CE3 (Carlson et al. 1995). The other possibility is a truncated core oligosaccharide that still serves as an acceptor for the intact O-antigen, known from Escherichia coli K8 strains (Whitfield 1995). However, as shown earlier, the mutated LPS was no longer recognized by a polyclonal antibody directed against the $S$. meliloti Rm2011 LPS (Lagares et al. 1992), indicating that the LPS part truncated in the case of the mutant Rm6963 exhibits the major antigenicity. Besides the LPS, other surface carbohydrates could be affected in the case of the mutant Rm6963. While the EPS I biosynthesis is not altered in Rm6963 we cannot exclude the possibility that the KDO-rich polysaccharide (Reuhs et al. 1993), or as-yet-unidentified compounds are altered. Further biochemical analysis is required to establish the function of the $\operatorname{lps} \mathrm{B}$ gene.

Most species of the closely related genera Medicago, Melilotus, and Trigonella form an effective symbiosis with S. meliloti (Provorov 1994). Nevertheless, a specific $S$. meliloti strain will not form an effective symbiosis with all species of Medicago, Melilotus, and Trigonella. For example, $S$. meliloti strains isolated from $M$. sativa are usually ineffective with $M$. arabica while strains isolated from $M$. arabica often form effective nodules with both species (Provorov 1994). It was therefore not surprising to find that S. meliloti Rm2011 and the LPS mutant Rm6963 formed an effective symbiosis with most but not all of the tested Medicago spp. Most interestingly, three species, namely, $M$. blancheana var. blancheana, M. truncatula, and M. truncatula var. truncatula, were not able to establish an effective symbiosis after inoculation with the LPS mutant Rm6963 while the wild-type strain Rm2011 was effective. A hostplant-dependent defect of LPS mutants was also observed for the broad-host-range Rhizobium GRH2 (Lopez-Lara et al. 1995). LPS mutants of this Acacia nodulating strain lost the ability to form effective nodules on Lotus corniculatus, Vicia hirsuta, Trifolium subterraneum, Trifolium repens, and Trifolium incarnatum while the symbiosis with Phaseolus vulgaris was not affected. To our knowledge, the mutant Rm6963 represents the first LPS mutant that alters the host range within one legume genus. The host-specific defect of

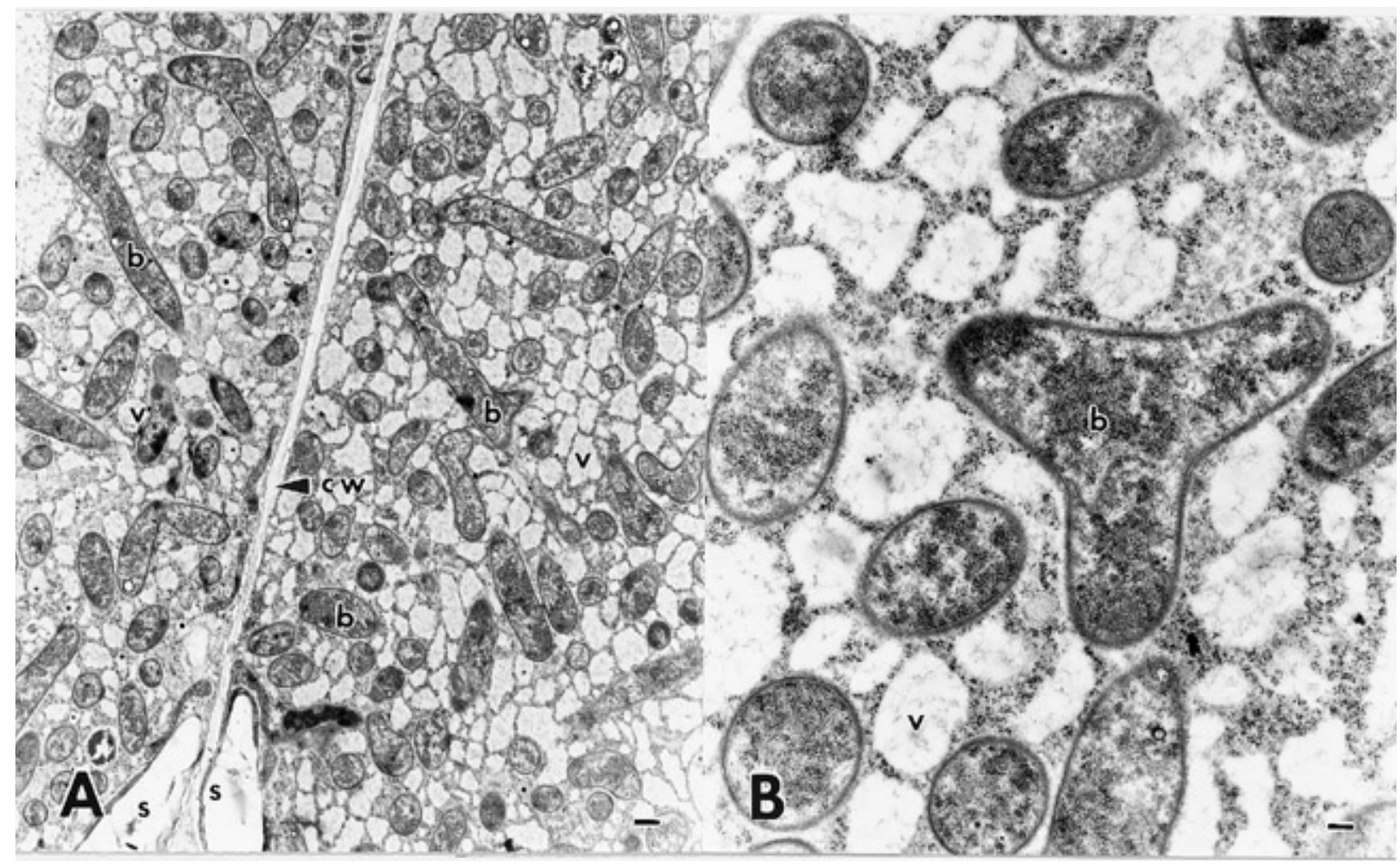

Fig. 4. Electron micrograph of the central tissue of a Medicago truncatula nodule induced by the Sinorhizobium meliloti lipopolysaccharide (LPS) mutant Rm6963. A, Infected plant cells within the symbiotic zone of a M. truncatula nodule induced by the S. meliloti LPS mutant Rm6963. B, Detailed view of bacteroids within the symbiotic zone. Plant cytoplasm is filled with small vesicle-like structures containing fibrillar material. b, bacteroids; cw, cell wall; s, stach granules; v, vesicles. Scale bar represents $1 \mu \mathrm{m}(\mathbf{A})$ and $0.25 \mu \mathrm{m}(\mathbf{B})$. 
the $S$. meliloti mutant Rm6963 could indicate a specific recognition of the rhizobial LPS rather than a nonspecific or structural function. Nevertheless, a direct effect of isolated LPS on cellular functions of the host plant remains to be shown. Although the mutant is deficient in nitrogen fixation on $M$. truncatula, the delay in nodulation was observed on $M$. sativa and $M$. truncatula. This might indicate that the LPS functions during the onset of nodulation and during the establishment of nitrogen-fixing nodules were specific. This hypothesis is strengthened by the observation that the surface antigens of $R$. leguminosarum change in a complex pattern during the infection and bacteroid development (Kannenberg et al. 1994), also indicating distinct functions during different steps in the establishment of symbiosis. Different LPS epitopes may be recognized in the various developmental stages of symbiosis. In addition, different host plants may recognize different parts of the LPS molecule. The delay in nodulation on $M$. sativa and M. truncatula indicated that both host plants recognized the change in the LPS structure. EPS mutants of $S$. meliloti are able to overcome the infection defect in low frequencies after a prolonged incubation (Niehaus et al. 1993). In contrast, effective nodules were never observed in the case of the $S$. meliloti LPS mutant Rm6963.

The microscopic analysis of root nodules induced by $S$. meliloti Rm6963 on M. truncatula revealed that this LPS mutant has a severe defect in nodule development with this par-

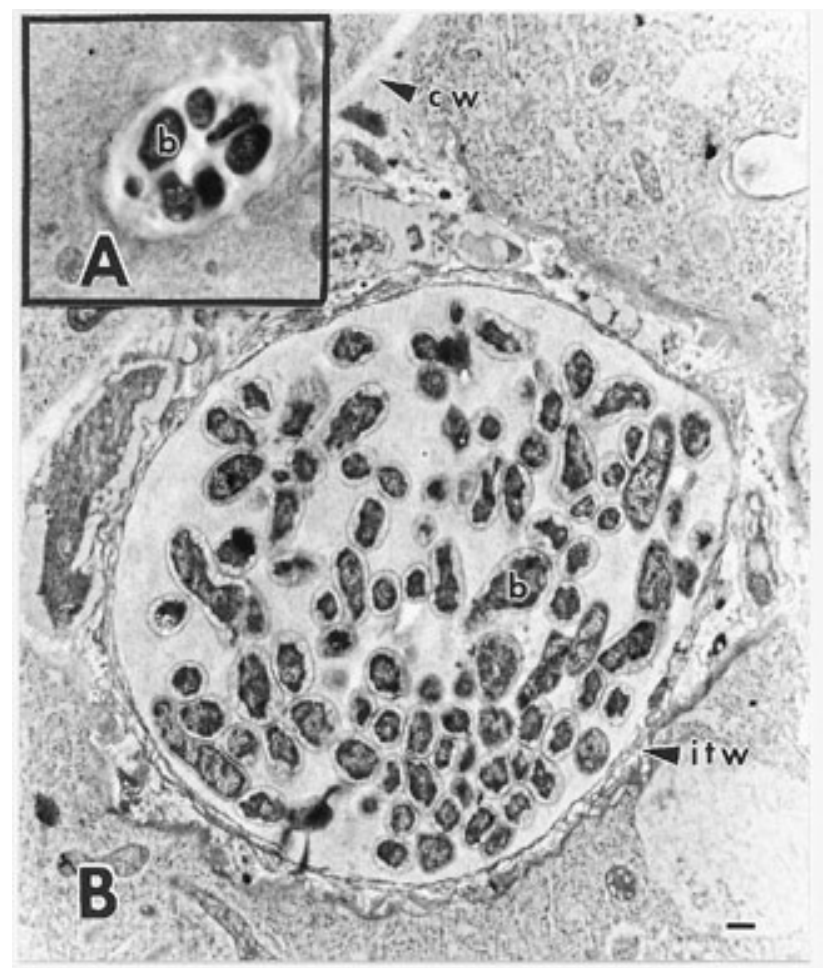

Fig. 5. Comparison of infection threads induced by the Sinorhizobium meliloti wild-type Rm2011 and the lipopolysaccharide (LPS) mutant Rm6963 within Medicago truncatula nodules. A, Infection thread within infection zone of an $M$. truncatula root nodule induced by the wild-type strain Rm2011. B, Infection thread within infection zone of an M. truncatula root nodule induced by the LPS mutant Rm6963 (at same magnification as in A). b, bacteria; cw, cell wall; itw, infection thread wall. Scale bar represents $1 \mu \mathrm{m}$. ticular host plant. As described for R. leguminosarum LPS mutants (DeMaagd et al. 1989; Priefer 1989) the induced nodules are small, white, and poorly infected. Besides some irregularly shaped bacteroids, the plant cells are filled with numerous vesicles. A similar observation was made by Stacey et al. (1991) for soybean root nodules induced by an LPSdefective mutant of $B$. japonicum. Interestingly, soybean nodules expressing an antisense cDNA, coding for the small GTP-binding protein $v r a b 7$, which is believed to be involved in vesicle transport and fusion, accumulated multiple vesicles in the infected plant cells (Cheon et al. 1993). From these data Verma and Hong (1996) hypothesized that the release of rhizobia from the infection thread and uptake into a symbiosome could follow a receptor-mediated endocytosis or integrinmediated phagocytosis process. The LPS could be involved in the induction of this process. The morphology of the infection threads was changed to the same extent in a characteristic manner on the light microscopic and ultrastructural level. Infection threads were enlarged and encrusted with cell wall material. Similar infection threads were formed by R. leguminosarum bv. viciae LPS mutants (Perotto et al. 1994) or during the delayed infection of $M$. sativa by EPS-deficient mutants of S. meliloti (Niehaus et al. 1993). In both cases, these changes in the infection thread morphology were interpreted as the consequence of a plant defense reaction against the symbiont. In accordance with this, autofluorescence of plant cells within the nodule was described after inoculation of pea plants with $R$. leguminosarum bv. viciae LPS mutants (Perotto et al. 1994). This typical plant defense reaction was also observed in this study. The LPS mutant Rm6963 induced an autofluorescence of the plant cells within the central tissue of the root nodule, in contrast to EPS-deficient $S$. meliloti mutants, in which the autofluorescence occurred in the cortical cell walls (Niehaus et al. 1993). In alfalfa cell cultures both the $S$. meliloti EPS and LPS are able to suppress defense-related responses (Niehaus et al. 1997). It seems that in the case of the S. meliloti / M. truncatula symbiosis distinct functions for EPS and LPS can be defined. EPS-deficient mutants failed to infect M. truncatula (Niehaus et al. 1994), while LPSdeficient mutants fail to colonize the plant cells after release from the infection threads. In both cases plant defense seems to be involved. We suppose that the $S$. meliloti EPS acts as a suppressor during the infection thread initiation while the LPS takes over this function after the release from the infection thread. Nevertheless, LPS may also have functions during the infection thread initiation since nodulation is delayed when plants are inoculated with the LPS mutant. This hypothesis is strengthened by the finding that preincubation of clover plants with $R$. leguminosarum bv. trifolii LPS promoted infection by $R$. leguminosarum bv. trifolii (Dazzo et al. 1991). This emphasizes the general importance of surface carbohydrates for this type of plant-microbe interaction. The $S$. meliloti LPS mutant Rm6963 effective in symbiosis with $M$. sativa but ineffective with $M$. truncatula denoted a specific recognition of the LPS by the host plant. Positive recognition via surface carbohydrates is well documented in different animal cell systems (Varik 1993). A central question for further work will be the proof of high affinity binding sides for rhizobial oligosaccharides in the host plants and the understanding of the signal transduction network making the decision for or against plant defense. 


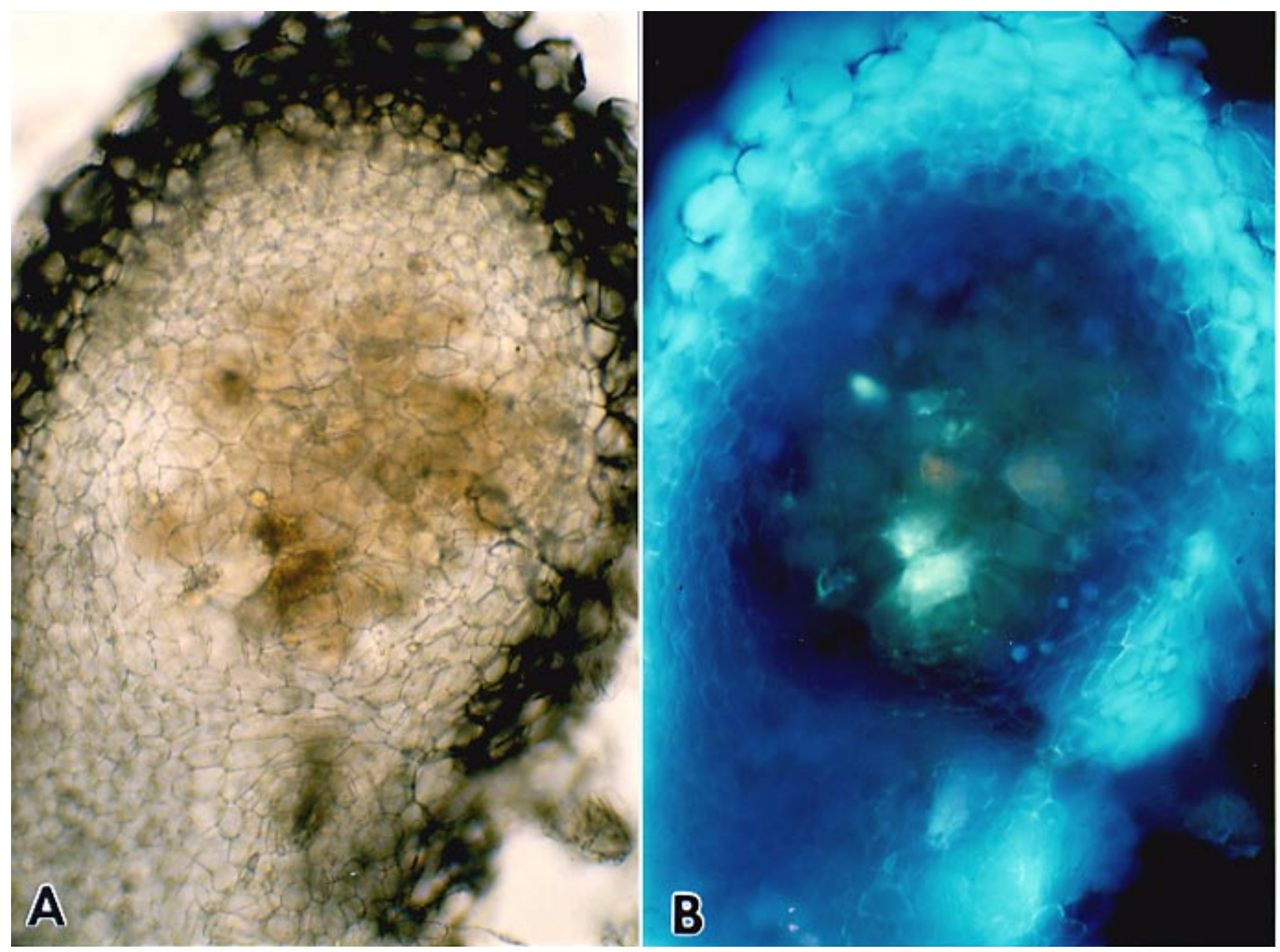

Fig. 6. Microscopic analysis of a hand section of a Medicago truncatula nodule induced by the Sinorhizobium meliloti mutant Rm6963. A, Section of total nodule viewed by bright field microscopy. Brown plant cells within central tissue became visible. B, Same section as in A, but visualized by blueviolet fluorescence microscopy.

\section{MATERIALS AND METHODS}

\section{Bacterial strains and plasmids.}

The E. coli and S. meliloti strains and the plasmids used in this study are listed in Table 2.

\section{Media and growth conditions.}

E. coli strains were cultivated at $37^{\circ} \mathrm{C}$ in Luria-Bertani (LB) medium (Maniatis et al. 1982). S. meliloti strains were grown in tryptone-yeast (TY) medium (in grams per liter: tryptone, $5 \mathrm{~g}$; yeast extract, $3 \mathrm{~g} ; \mathrm{CaCl}_{2}, 0.4 \mathrm{~g}$ ) at $30^{\circ} \mathrm{C}$. Antibiotics were added at the following concentrations (per liter): $600 \mathrm{mg}$ of streptomycin; $100 \mathrm{mg}$ of neomycin; $50 \mathrm{mg}$ of gentamicin; and $5 \mathrm{mg}$ of tetracycline for S. meliloti; and $100 \mathrm{mg}$ of neomycin; $10 \mathrm{mg}$ of gentamicin; and $10 \mathrm{mg}$ of tetracycline for E. coli.

\section{DNA biochemistry.}

DNA isolation, restriction enzyme analysis, and cloning procedures were performed by established techniques (Maniatis et al. 1982). Enzymes were purchased from Pharmacia (Freiburg, Germany).

\section{Construction of plasmid pJL201.}

Total DNA of the mutant Rm6963 was isolated and digested with SstI. The digest was cloned into the vector pSVB30 and transformed into E. coli JM83. Neomycin resistant clones carrying the Tn5-marked DNA region were obtained. This $S$. meliloti DNA fragment was subcloned in pACYC-Gm ${ }^{\mathrm{r}} \mathrm{mob}$ (pJL201) and transformed into E. coli S17.1. Fragmentspecific transposon mutagenesis was achieved by introducing pJL201 into S. meliloti Rm2011 and selection for the neomycin resistance of the Tn5 and absence of the tetracycline resistance of the vector plasmid. The resulting Tn5 mutant was termed RmJL1.

\section{Construction of plasmid pAL101.}

The plasmid integrated into the strain RmJL1 was able to excise spontaneously at a low frequency, together with the wild-type or the mutated $S$. meliloti DNA fragment. With the helper plasmid RP4-4-7, the excised plasmid was transferred back to $E$. coli. Clones carrying the wild-type fragment were isolated by selection for those clones exhibiting the antibiotic resistance of the plasmid but missing the $\mathrm{Km}$ resistance of the Tn5. 
Table 2. Microbial strains and plasmids used in this study

\begin{tabular}{|c|c|c|}
\hline Name & Relevant characteristics & Source \\
\hline \multicolumn{3}{|l|}{ Sinorhizobium meliloti } \\
\hline $\mathrm{Rm} 2011$ & Wild type & Cassé et al. 1979 \\
\hline Rm6963 & Lipopolysaccharide-deficient $\operatorname{Tn} 5$ mutant & Lagares et al. 1992 \\
\hline $\operatorname{Rm} 7670$ & $\operatorname{lps} \mathrm{B}:: \operatorname{Tn} 5$ & Clover et al. 1989 \\
\hline $\operatorname{Rm} 7555$ & lps $\mathrm{C}:: \operatorname{Tn} 5$ & Clover et al. 1989 \\
\hline RmJL1 & Tn5::5-kb SstI fragment in Rm2011 & This work \\
\hline \multicolumn{3}{|l|}{ Escherichia coli } \\
\hline JM83 & $\mathrm{d}$ lac-pro, $\mathrm{Sm}^{\mathrm{r}}$ & Vieira and Messing 1982 \\
\hline S17-1 & RP4 derivative integrated & Priefer et al. 1985 \\
\hline \multicolumn{3}{|l|}{ Plasmids } \\
\hline pSVB30 & Sequencing vector with mcs & Priefer et al. 1985 \\
\hline pACYC-Gmrmob & & Arnold and Pühler 1988 \\
\hline pJL201 & pACYC-Gm ${ }^{\mathrm{r}} \mathrm{mob}$ and $\mathrm{Tn} 5:: 5-\mathrm{kb}$ SstI fragment & This work \\
\hline pAL101 & pACYC-Gm ${ }^{\mathrm{r}} \mathrm{mob}$ and $5-\mathrm{kb}$ SstI fragment & This work \\
\hline pRP4-4-7 & RP4 derivative used for mobilization & Priefer et al. 1985 \\
\hline
\end{tabular}

\section{SDS-PAGE of LPS.}

For SDS-PAGE, the LPS was solubilized from proteinase $\mathrm{K}$ treated cells (Goldman and Leive 1987). The pellet from $1 \mathrm{ml}$ of bacterial suspension of an overnight culture was lysed by heating in a boiling water bath in $125 \mu \mathrm{l}$ of $60 \mathrm{mM}$ Tris- $\mathrm{HCl}$ (pH 6.8)-1\% SDS for $5 \mathrm{~min}$ and then diluted to $500 \mu \mathrm{l}$ with the same buffer without SDS. Benzonase (200 U; Sigma, Deisenhofen, Germany) was added and incubated at $37^{\circ} \mathrm{C}$ for $4 \mathrm{~h}$ to destroy DNA and RNA contaminations. Proteinase K was added to a final concentration of $10 \mu \mathrm{g} / \mathrm{ml}$, and incubated for a further $24 \mathrm{~h}$. Electrophoresis was done with a $16.5 \%$ polyacrylamide gel with the tricine buffer system as described by Lesse et al. (1990). LPS reference samples from $S$. typhimurium (smooth strain), TV119 (Ra mutant), and SL1181 (Re mutant) were obtained from Sigma. After electrophoresis, the gels were fixed and silver stained as described by Tsai and Frasch (1982).

\section{Plant test and determination of nitrogen fixation.}

The plant test was carried out as described by Rolfe et al. (1980). Plants were grown on a synthetic, nitrogen-free agar medium. Each petri dish containing four seedlings was inoculated with $0.1 \mathrm{ml}$ of an $S$. meliloti strain cultured overnight. For measurements of acetylene reduction activity of different Medicago spp., 10 nodulated plants were taken and tested individually as described by Somasegaran and Hoben (1985). Plants fixing less than $4 \%$ of the wild-type inoculated control were defined as Fix ${ }^{-}$. For comparison of the acetylene reduction activity of $M$. sativa $\mathrm{cv}$. Du Puits with $M$. truncatula, 10 plants were taken at different time intervals. The absolute amount of acetylene reduction was estimated by comparison with an acetylene standard (Linde, Höllriegelskreuth, Germany). Total nitrogen in dry mass was estimated from five samples each containing four plants, by the microKjeldahl method.

\section{Microscopy.}

Nodules were harvested 3 weeks after inoculation, placed in fixative, cut longitudinally, and fixed for $2 \mathrm{~h}$ on ice in $2.5 \%$ glutaraldehyde in $0.1 \mathrm{M}$ sodium cacodylate buffer $(\mathrm{pH} 7.2)$. They were then dehydrated in graded ethanol series, infiltrated, and embedded in LR-White resin (Plano, Marburg, Germany). Semi-thin and ultra-thin sectioning, uranyl acetatelead staining, and microscopy were carried out as described by
Kapp et al. (1990). Fluorescence microscopy was carried out as described by Niehaus et al. (1993).

\section{ACKNOWLEDGMENTS}

This work was supported by a grant of the Deutsche Forschungsgemeinschaft (Ni 474/1-3). We thank K. Hammer, Gatersleben (FRG) for the kind provision of Medicago seeds and D. Jording for critical reading of the manuscript.

\section{LITERATURE CITED}

Arnold, W., and Pühler, A. 1988. A family of high-copynumber plasmid vectors with single end-label sites for rapid nucleotide sequencing. Gene 70:171-179

Brewin, N. J. 1991. Development of the legume root nodule. Annu. Rev. Cell Biol. 7:191-226.

Carlson, R. W. 1984. Heterogenicity of Rhizobium lipopolysaccharides. J. Bacteriol. 158:548-555.

Carlson, R. W., Forsberg, L. S., Price, N. P., Bhat, U. R., Kelly, T. M., and Raetz, C. R. 1995. The structure and biosynthesis of Rhizobium leguminosarum lipid A. Prog. Clin. Biol. Res. 392:25-31.

Carlson, R. W., Kalembasa, S., Turowski, D., Pachori, P., and Noel, K. D. 1987. Characterization of the lipopolysaccharide from a Rhizobium phaseoli mutant that is defective in infection thread development. J. Bacteriol. 169:4923-4928.

Carlson, R. W., and Krishnaiah, B. S. 1992. Structures of the oligosaccharides obtained from the core regions of the lipopolysaccharides of Bradyrhizobium japonicum 61A101c and its symbiotically defective lipopolysaccharide mutant, JS314. Carbohydr. Res. 231:205-219.

Cassé, F., Boucher, C., Julliot, J. S., Michel, M., and Denarie, J. 1979. Identification and characterization of large plasmids in Rhizobium meliloti using agarose gel electrophoresis. J. Gen. Microbiol. 113: 229-242.

Cheon, C. C., Lee, N.-G., Siddique, A.-B. M., Bal, A. K., and Verma, D. P. S. 1993. Roles of plant homologs of Rab1p and Rab7p in the biogenesis of the peribacteroid membrane, a subcellular compartment formed de novo during root nodule symbiosis. EMBO J. 12:41254135

Clover, R. H., Kieber, J., and Signer, E. R. 1989. Lipopolysaccharide mutants of Rhizobium meliloti are not defective in symbiosis. J. Bacteriol. 171:3961-3967.

Dazzo, F. B., Truchet, G. L., Hollingsworth, R. I., Hrabak, E. M., Pankratz, H. S., Philip-Hollingsworth, S., Salzwedel, J. L., Chapman, K., Appenzeller, L., and Squartini, A. 1991. Rhizobium lipopolysaccharide modulates infection thread development in white clover root hairs. J. Bacteriol. 173:5371-5384.

DeMaagd, R. A., Rao, A. S., Mulders, I. H. M., Goosen-de Roo, L., VanLoosdrecht, M. C. M., Wijffelman, C. A., and Lugtenberg, B. J. J. 1989. Isolation and characterization of mutants of Rhizobium leguminosarum bv. viciae 248 with altered lipopolysaccharides: Possible role of surface charge or hydrophobicity in bacterial release from the in- 
fection thread. J. Bacteriol. 171:1143-1150.

Finan, T. M., Hirsch, A. M., Leigh, J. A., Johansen, E., Kuldau, G. A., Deegan, S., Walker, G. C., and Signer, E. R. 1985. Symbiotic mutants of Rhizobium meliloti that uncouple plant from bacterial differentiation. Cell 40:869-877.

Goldman, R. C., and Leive, L. 1987. Electrophoretic separation of lipopolysaccharide monomeres differing in polysaccharide length. Methods Enzymol. 138:267-275.

Hirsch, A. 1992. Developmental biology of legume nodulation. New Phytol. 122:211-237.

Hitchcock, P. J., and Brown, T. M. 1983. Morphological heterogenicity among Salmonella lipopolysaccharide chemotypes in silver-stained polyacrylamide gels. J. Bacteriol. 154:269-277.

Kannenberg, E. L., and Brewin, N. J. 1994. Host-plant invasion by Rhizobium: The role of cell-surface components. Trends Microbiol. 2: 277-283.

Kannenberg, E. L., Perotto, S., Bianciotto, V., Rathbun, E. A., and Brewin, N. J. 1994. Lipopolysaccharide epitope expression of Rhizobium bacteroids as revealed by in situ immunolabelling of pea root nodule sections. J. Bacteriol. 176:2021-2032.

Kapp, D., Niehaus, K., Quandt, J., Müller, P., and Pühler, A. 1990. Cooperative action of Rhizobium meliloti nodulation and infection mutants during the process of forming mixed infected alfalfa nodules. Plant Cell 2:139-151.

Lagares, A., Caetano Anolles, G., Niehaus, K., Lorenzen, J., Ljunggren, H. D., Puhler, A., and Favelukes, G. 1992. A Rhizobium meliloti lipopolysaccharide mutant altered in competitiveness for nodulation of alfalfa. J. Bacteriol. 174:5941-5952.

Leigh, J. A., and Coplin, D. L. 1992. Exopolysaccharides in plantbacterial interactions. Annu. Rev. Microbiol. 46:307-346.

Lesse, A. J., Campagnari, A. A., Bittner, W. E., and Apicella, M. A. 1990. Increased resolution of lipopolysaccharides and lipooligosaccharides utilizing tricine-sodium dodecyl sulfate-polyacrylamide gel electrophoresis. J. Immunol. Methods 126:109-117.

Lopez-Lara, I. M., Orgambide, G., Dazzo, F. B., Olivares, J., and Toro, N. 1995. Surface polysaccharide mutants of Rhizobium sp. (Acacia) strain GRH2: Major requirement of lipopolysaccharide for successful invasion of Acacia nodules and host range determination. Microbiology 141:573-581.

Maniatis, T. A., Fritsch, E. F., and Sambrook, J. 1982. Molecular Cloning: A Laboratory Manual. Cold Spring Harbor Laboratory, Cold Spring Harbor, NY.

Müller, P., Hynes, M. F., Kapp, D., Niehaus, K., and Pühler, A. 1988. Two classes of Rhizobium meliloti infection mutants differ in exopolysaccharide production and in coinoculation properties with nodulation mutants. Mol. Gen. Genet. 211:17-26.

Niehaus, K., Baier, R., Kohring, B., Flaschel, E., and Pühler, A. 1997. Symbiotic suppression of the Medicago sativa plant defense system by Rhizobium meliloti oligosaccharides. Pages 111-114 in: Biological Fixation of Nitrogen for Ecology and Sustainable Agriculture. A. Legocki,
H. Bothe, and A. Pühler, eds. NATO ASI series. Springer, Heidelberg.

Niehaus, K., Baier, R. Lorenzen, J., Meyer-Gattermann, P., Sieben, S., and Pühler, A. 1994. Plant defense in alfalfa pseudonodules induced by an exopolysaccharide (EPS I)-deficient symbiont Rhizobium meliloti. Acta Hortic. 381:258-264.

Niehaus, K., Kapp, D., and Pühler, A. 1993. Plant defense and delayed infection of alfalfa pseudonodules induced by an exopolysaccharide (EPS I)-deficient Rhizobium meliloti mutant. Planta 190:415-425.

Noel, D. K. 1992. Rhizobial polysaccharides required in symbiosis with legumes. Pages 341-357 in: Molecular Signals in Plant-Microbe Communication. D. P. Verma, ed. CRC Press, Boca Raton, FL.

Perotto, S., Brewin, N. J., and Kannenberg, E. L. 1994. Cytological evidence for a host defense response that reduces cell and tissue invasion in pea nodules by lipopolysaccharide-defective mutants of Rhizobium leguminosarum strain 3841. Mol. Plant-Microbe Interact. 7:99-112.

Priefer, U. B. 1989. Genes involved in lipopolysaccharide production and symbiosis are clustered on the chromosome of Rhizobium leguminosarum biovar viciae VF39. J. Bacteriol. 171:6161-6168.

Priefer, U., Simon, R., and Pühler, A. 1985. Extension of the host range of Escherichia coli vectors by incorporation of RSF1010 replication and mobilization functions. J. Bacteriol. 163:324-330

Provorov, N. A. 1994. The independence between taxonomy of legumes and specificity of their interaction with rhizobia in relation to evolution of the symbiosis. Symbiosis 17:183-200.

Reuhs, B. L., Carlson, R. W., and Kim, J. S. 1993. Rhizobium fredii and Rhizobium meliloti produce 3-deoxy-D-manno-2-octulosonic acid containing polysaccharides that are structurally analogous to group II K-antigens (capsular polysaccharides) found in Escherichia coli. J. Bacteriol. 175:3570

Rolfe, B. G., Gresshoff, P. M., and Shine, J. 1980. Rapid screening for symbiotic mutants of Rhizobium and white clover. Plant Sci. Lett. 19: 277-284.

Somasegaran, P., and Hoben, H. J. 1985. Methods in legume-Rhizobium technology. Paia Maui, Hawaii, NifTAL Project.

Stacey, G., So, J.-S., Roth, L. E., Lakshmi S. K., B., and Carlson, R. W. 1991. A lipopolysaccharide mutant of Bradyrhizobium japonicum that uncouples plant from bacterial differentiation. Mol.-Plant-Microbe Interact. 4:332-340.

Tsai, C. M., and Frasch, C. F. 1982. A sensitive silver stain for detecting lipopolysaccharides in polyacrylamide gels. Anal. Biochem. 119:115119.

Varik, A. 1993. Biological roles of oligosaccharides: All of the theories are correct. Glycobiology 3:97-130.

Verma, D. P. S., and Hong, Z. 1996. Biogenesis of the peribacteroid membrane in root nodules. Trends Microbiol. 4:364-368

Vieira, J., and Messing, J. 1982. The pUC plasmids, an M13mp7derived system for insertion mutagenesis and sequencing with synthetic universal primers. Gene 19:259-268.

Whitfield, C. 1995. Biosynthesis of lipopolysaccharide O-antigens. Trends Microbiol. 3:178-185. 